\title{
The Effect of Natural Health Products and Traditional Medicines on the Activity of Human Hepatic Microsomal-Mediated Metabolism of Oseltamivir
}

\author{
Rui Liu ${ }^{1,2}$, Teresa W. Tam ${ }^{1,2}$, Jingqin $\mathrm{Mao}^{2}$, Ammar Saleem $^{2}$, Anthony Krantis ${ }^{1,2}$, John T. Arnason ${ }^{2}$, Brian C. Foster ${ }^{1,2,3}$ \\ ${ }^{1}$ Department of Cellular and Molecular Medicine, University of Ottawa, Ottawa, ON, Canada \\ ${ }^{2}$ Centre for Research in Biopharmaceuticals and Biotechnology, University of Ottawa, Ottawa, ON, Canada \\ ${ }^{3}$ Therapeutic Products Directorate, Health Canada, Ottawa, ON, Canada.
}

Received, October 26, 2009; Revised, February 3, 2010; Accepted, February 9, 2010; Published, February 17, 2010.

\begin{abstract}
Purpose. Oseltamivir is a prodrug that requires metabolic activation but there is little information on whether natural health products interact to prevent the biotransformation by the carboxylesterase. Methods. HPLCDAD-ESI-MSD and fluorometric assays were used to determine if 50-pooled mixed gender human liver microsomes can mediate the formation of the active carboxylate metabolite and then if this reaction is affected by natural health products. Results. Extracts from 6 traditional Cree botanicals, a commercially available Echinacea product, Goldenseal and a traditional Chinese medicine reduced the formation of the active drug. In addition to oseltamivir carboxylate we report the detection of two new metabolites which are derivatives of oseltamivir carboxylate, one of which is a metabonate formed as a result of methanol. Conclusions. In vitro studies would suggest that there is the potential for some natural health products used by patients in response to pandemic $\mathrm{A} / \mathrm{H} 1 \mathrm{~N} 1$ to reduce drug efficacy. Further studies are required to determine if these potential interactions could be clinically significant.
\end{abstract}

\section{INTRODUCTION}

Oseltamivir (GS4104, Ro-64-0796) is an anti-viral prodrug that requires metabolic activation through carboxylesterase 1-mediated biotransformation to the active oseltamivir carboxylate (GS4071) metabolite for the prevention and treatment of uncomplicated acute illness due to influenza infection $(1,2)$. Human carboxylesterase 1 is highly expressed in hepatic tissue. The human carboxylesterase 1 (CES1) gene is polymorphic with a nonconservative substitution at codon 143 (p.Gly143Glu) and a frameshift mutation at codon 260 (p.Asp260fs) (3). Codon 143 frequency ranged between $3.7 \%, 4.3 \%, 2.0 \%$ and $0 \%$ in the white, black, Hispanic and Asian populations examined, respectively. In vitro testing suggests that the catalytic function of both polymorphisms is substantially impaired and substrate-dependant. Other products such as aspirin, clopidogrel, deltamethrin and permethrin are also metabolized by this enzyme (2). The carboxylate metabolite is excreted into the urine by the organic anion transporter (4).

Oseltamivir, but not the carboxylate metabolite is a substrate for the P-glycoprotein efflux transporter which may limit absorption and transport across various blood barriers (5). Uptake of oseltamivir by Caco-2 and HeLa cells transfected with peptide transporter1 (HeLa/PEPT1) was timeand temperature-dependent (6). This uptake by Caco-2 cells and HeLa/PEPT1 was saturable, with similar $\mathrm{Km}$ values. Intestinal absorption of oseltamivir in adult rats was greatly reduced by simultaneous administration of milk, casein or by the PEPT1 inhibitor glycyl-sarcosine. PEPT1 is also known to transport $\beta$-lactam antibiotics, anti-viral and anti-cancer agents.

Corresponding Author: Brian C. Foster, Health Canada, Therapeutic Products Directorate, Holland Cross A.L., 1600 Scott Street, Ottawa, ON, Canada. Email: brian_foster@hcsc.gc.ca 
In a limited examination of 104 human liver samples, there was a large inter-individual variability in the mRNA (430 fold), protein (100 fold) and hydrolytic activity towards oseltamivir (127 fold) (2). The foetal (82 to 224 gestation days) hydrolytic activity towards oseltamivir was less than $10 \%$ of the adult (greater than 18 years of age) level. The neonatal-children ( 0 day to 10 years) grouping was approximately $25 \%$ of the adult level. The CES1 form had a much greater inter-group and inter-individual variation than the CES2 form. CES1 had 319-fold higher mRNA expression in the adult compared with the foetal group. In adults, there was a 430 -fold range in the inter-individual mRNA. Zhu et al. (7) also noted that the average CES1 expression in the subjects $<1$ year of age was significantly lower than that of pooled samples. CES1 expression in the 13 day and 1 month-old subjects were $20.3 \%$ and $11.1 \%$, respectively, of the pooled sample values (11). Mouse hepatic CES1 expression and activity was markedly lower in newborns relative to adults, and increased gradually with age, approximating levels of adult animals by age 2 to 4-weeks (11). No significant differences of the expression and activity of CES1 were observed between sexually mature male and female mice. The effect of gender in humans on this enzyme is not known. Exposure to the active metabolite at steady state is approximately $25 \%$ higher in elderly patients $(>$ or $=65$ years) compared with younger individuals. The active metabolite was rapidly formed and present in plasma at significantly higher and longer lasting amounts than those of parent substrate $(4,8)$. Pharmacokinetics of both compounds was linear.

In addition to formation of the active drug, oseltamivir should be metabolized through glucuronidation, but these metabolites have not been reported (4). In rat, a minor route of biotransformation appears to be through $\omega$ hydroxylation of the ethylpropyloxy side chain to (R)- and (S)- $\omega$-hydroxylation products (9). The predominate $(\mathrm{R})-\omega$-hydroxylation product is then oxidized to the (R)- $\omega$-carboxylate (M3).

The potential for food other than the milk and casein effect on PEPT1-mediated transport in rat, and natural health product interactions with oseltamivir is not known. Any interaction that may either reduce the amount of prodrug available or inhibit the biotransformation to the active drug may impact the safety and efficacy of this product.

In this study we examined the human hepatic microsomal-mediated metabolism of oseltamivir in the presence of several natural health products including Cree traditional medicines. Assisted by LC-DAD-ESI we examined the metabolite profile to follow conversion of 4 to a well known metabolite 2 and formation of a novel metabonate 3.

\section{METHODS}

\section{Chemicals and Plant Material}

HPLC grade methanol (MeOH), ethyl acetate, and acetonitrile were purchased from Fisher Scientific (Ottawa, ON, Canada). Dibenzylfluorescein (DBF), 7-benzyloxy-4-(trifluoromethyl) coumarin (BFC), and 50-pooled mixed gender human liver microsomes (HLM) were purchased from BD Biosciences (Mississauga, ON, Canada). Nicotinamide adenine dinucleotide phosphate, reduced form (NADPH) was purchased from Sigma-Aldrich (Oakville, ON, Canada). Ketoconazole was purchased from Calbiochem (Gibbstown, NJ, USA). Oseltamivir phosphate and oseltamivir carboxylate were purchased from Toronto Research Chemicals Inc. (North York, ON, Canada).

The Cree botanical samples (Table 1) were harvested in Mistissini, Quebec, Canada following the instructions of the elders and healers of this community (15). Plants were identified by Dr. A. Cuerrier and voucher specimens were deposited at the Marie-Victorin herbarium at the Montreal Botanic garden, Montreal, Quebec, Canada. Airdried samples were washed and separated by organ parts. The appropriate part was ground using a Wiley Mill with a $2 \mathrm{~mm}$ filter (A.H. Thomas Co., Swedesboro, N.J., USA) and extracted twice with $80 \%$ ethanol $(10 \mathrm{~mL} / \mathrm{gm})$ for $24 \mathrm{hr}$. The extracts were combined, filtered and reduced to dryness through rotary evaporation and then lyophilization (16). The plant extracts were solubilized in $95 \%$ ethanol for testing. 
Table 1. List of natural health products and traditional Cree botanicals and their traditional medicinal uses.

\begin{tabular}{|c|c|}
\hline Name & Medicinal Use \\
\hline Chrysanthemum Flower, Natural Research Pprogramme (NRP) 265 & $\begin{array}{l}\text { Anti-inflammatory, antimicrobial, prevents } \\
\text { cardiopathy. }\end{array}$ \\
\hline Isatis Root, NRP 267 & $\begin{array}{l}\text { Reduces fever, detoxificant, prevents virulent } \\
\text { cold, swollen pharynx and larynx }\end{array}$ \\
\hline Chai Hu, NRP 269 & $\begin{array}{l}\text { Treats common cold, fever, cough, infectious } \\
\text { hepatitis, liver cirrhosis, erythema and globus } \\
\text { hystericus }\end{array}$ \\
\hline Goldenseal, ground root, NRP 273 & $\begin{array}{l}\text { Anti-catarrhal, anti-inflammatory, antiseptic, } \\
\text { astringent, bitter tonic, laxative, and } \\
\text { muscular stimulant }\end{array}$ \\
\hline $\begin{array}{l}\text { Echinacea (Echinacea purpurea), } 500 \mathrm{mg} \text { powder capsules, aerial } \\
\text { parts of the plant, NRP } 451\end{array}$ & Prevents common cold, anti-tumorogenic \\
\hline Panax quinquefolium extract, capsules, NRP 452 & Treats common cold, fever \\
\hline Abies balsamea (L.) Mill., Innasht & Sacred knowledge \\
\hline Alnus incana ssp. Rugosa (Du Roi) Clausen, Atushpi & Sacred knowledge \\
\hline Gaultheria hispidula (L.) Muhl., Pieuminaan & Sacred knowledge \\
\hline Juniperus communis L., Kahkachiminahtikw & Sacred knowledge \\
\hline Kalmia angustifolia L., Uishichipukw; & Sacred knowledge \\
\hline Larix laricina K.Koch, Watnagan & Sacred knowledge \\
\hline Lycopodium clavatum L., Pashtanhoagin & Sacred knowledge \\
\hline Picea mariana (Mill.) BSP., Innahtikw & Sacred knowledge \\
\hline Picea glauca (Moench) Voss, Minhikw & Sacred knowledge \\
\hline Pinus banksiana Lamb., Ushchishk & Sacred knowledge \\
\hline Populus balsamifera L., Mitus & Sacred knowledge \\
\hline Rhododendron groenlandicum (Oeder) Kron \& Judd, Kachichpukw & Sacred knowledge \\
\hline $\begin{array}{l}\text { Rhododendron tomentosum (Stokes) Harmaja ssp. subarcticum } \\
\text { (Harmaja) G. Wallace, Wishichipikushh }\end{array}$ & Sacred knowledge \\
\hline Salix planifolia Pursh, Pieuatikw & Sacred knowledge \\
\hline Sarracenia purpurea L., Ayigadash & Sacred knowledge \\
\hline Sorbus decora (Sarg.) Schneid., Mushkuminanatikw & Sacred knowledge \\
\hline Vaccinium vitis-ideae L., Wishichimna & Sacred knowledge \\
\hline
\end{tabular}

Other natural products were obtained from local sources and assigned unique identifying Nutraceutical Research Programme (NRP) accession numbers. Product vouchers were stored in the herbarium at the University of Ottawa. All formulated products were tested within their expiry date. NHPs 265, 267 and 269 were obtained as sachets and made to a $20 \mathrm{mg} / \mathrm{mL}$ water stock solution; a $5 \mu \mathrm{L}$ aliquot was used for testing. A stock solution of $20 \mathrm{mg} / \mathrm{mL}$ ethanol of Goldenseal (NRP 273) was diluted to $1 \mathrm{mg} / \mathrm{mL}$ by water before tested; a $5 \mu \mathrm{L}$ aqueous aliquot was used for testing. Traditional Labrador tea or Juniper tea was prepared by adding $1 \mathrm{gm}$ of dried material to 250 $\mathrm{mL}$ of boiling water. The water was maintained at a boiling temperature and aliquots of the tea were collected after 20 or $60 \mathrm{~min}$ of boiling.

\section{CYP inhibition assay}

The assay was performed in triplicate in 96-well plates with white walls and clear, flat bottoms under red-colored light to minimize the exposure of fluorescent light to photosensitive material. The fluorescence was measured using a Cytofluor 4000 Fluorescence Measurement System (Applied Biosystems, Foster City, CA). For the substrate $\mathrm{DBF}$, a solution consisting of $10 \mu \mathrm{M}$ oseltamivir, sample extract or control vehicle, $20 \mu \mathrm{g}$ HLM, 10 DBF (dissolved in acetonitrile) and $0.6 \mathrm{mM}$ NADPH in $185 \mathrm{mM}$ phosphate buffer solution (PBS, pH 7.5) buffer was incubated at $37^{\circ} \mathrm{C}$ for 30 min. The initial and final fluorescence was read at $485 \mathrm{~nm}$ excitation and $530 \mathrm{~nm}$ emission with a gain of 45. For the substrate BFC, a solution consisting 
of $30 \mu \mathrm{M}$ oseltamivir, $50 \mu \mathrm{g}$ HLM, $30 \mu \mathrm{M}$ BFC (dissolved in acetonitrile) and 0.6 mM NADPH in $100 \mathrm{mM}$ phosphate buffer solution (buffer, $\mathrm{pH}$ 7.4) was incubated at $37^{\circ} \mathrm{C}$ for $40 \mathrm{~min}$. The initial and final fluorescence was read at $406 \mathrm{~nm}$ excitation and $530 \mathrm{~nm}$ emission with a gain of 75 . The positive inhibitor used was $1.0 \mu \mathrm{M}$ ketoconazole (dissolved in $\mathrm{MeOH}$ ). Initial fluorescence was subtracted from respective final fluorescence for the calculations. The percent inhibition of each extract was calculated relative to the HLM activity with the vehicle control.

\section{CES-1 Inhibition Assay}

\section{HPLC}

HLM were thawed in $37^{\circ} \mathrm{C}$ water bath, and then placed on ice until required. A $5 \mu 1$ sample aliquot of the test extract was incubated in either $50 \mathrm{mM}$ Tris (pH 7.4) with $2 \mathrm{mg} / \mathrm{mL}$ HLM and $1.6 \mathrm{mM}$ oseltamivir for $30 \mathrm{~min}$ in a $37^{\circ} \mathrm{C}$ water bath with appropriate vehicle controls. The reaction was stop by $200 \mu \mathrm{L}$ of cold acetonitrile, the mixture was then centrifuged at $12,000 \mathrm{rpm}$ for $5 \mathrm{~min}$ and the supernatant fluid was filtered through 0.2 micron PTFE disc filters (Chromatographic Specialties Inc, Brockville, ON, Canada) before analysis.

An Agilent 1100 Series HPLC-DAD was used to monitor the reaction. A $15 \mu \mathrm{L}$ extract of reaction mixture was injected into a Supelco LC-BD C18 column $(5 \mu \mathrm{M}$ practical size, $250 \mathrm{mM} \times 4.6 \mathrm{~mm}$; Supelco, Ottawa, ON, CA) and separated by a gradient eluted method initial with a ratio of acetonitrile- $0.1 \%$ trifluoroacetic acid at 15:85 (v/v) for $2 \mathrm{~min}$ to $60: 40(\mathrm{v} / \mathrm{v})$ at $7 \mathrm{~min}$ with a flow rate of $1.2 \mathrm{~mL} / \mathrm{min}$, column temperature was set at $35^{\circ} \mathrm{C}$ and the DAD was set at $230 \mathrm{~nm}$.

\section{HPLC-DAD-APCI-MSD}

HLM were incubated and prepared as above. Analysis was undertaken by injecting $20 \mu \mathrm{L}$ through autosampler into an Agilent 1100 series HPLC-DAD-ESI-MSD analytical system (Agilent Technologies, Santa Ana, CA, USA). The system consisted of an autosampler (with a $100 \mu \mathrm{L}$ built in loop),

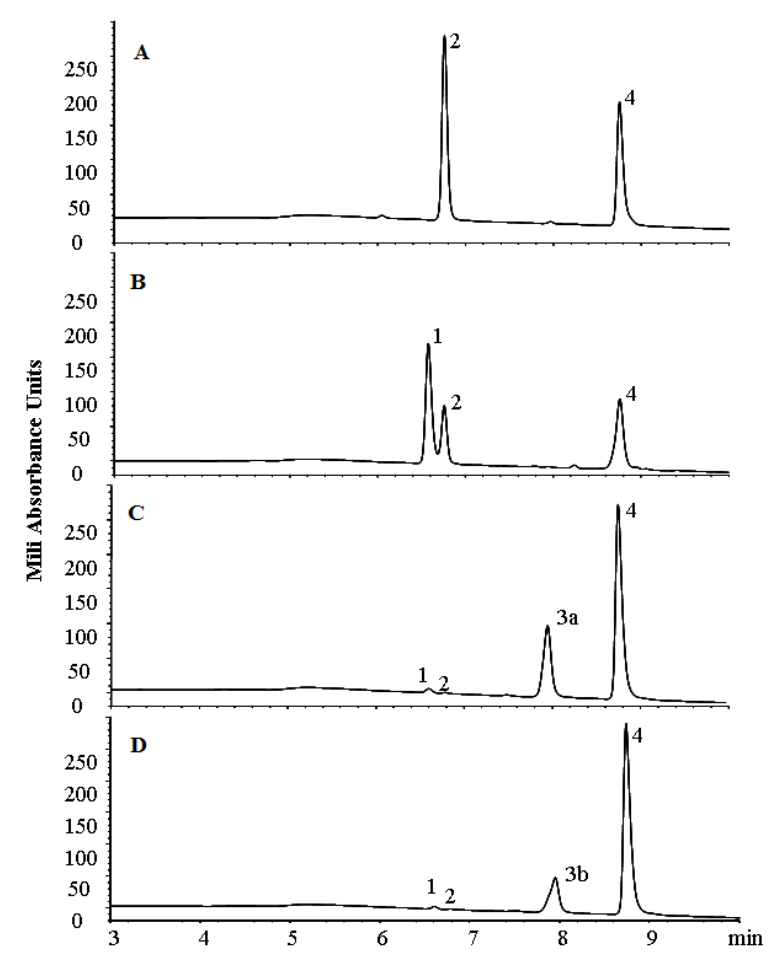

Figure 1. HPLC-DAD profiles of authentic standards and oseltamivir phosphate incubated under different incubation conditions: (A) authentic oseltamivir carboxylate (2) and oseltamivir phosphate (4); human liver microsome incubation in Tris buffer ( $\mathrm{pH}$ 7.4) with oseltamivir phosphate (4) prepared in $\mathrm{H}_{2} \mathrm{O}(\mathrm{B})$, in methanol (C) and $\mathrm{CD}_{3} \mathrm{OD}(\mathrm{D})$. 
a quaternary pump (maximum pressure limit of 400 bars), a column thermostat, a photodiode array detector, an ESI-MSD with a mass range of 100$1500 \mathrm{amu}$. The Chemstation software (version B.03.01) was used for the acquisition and data analysis. For the analysis authentic oseltamivir and its metabolite were injected as a mixture into the analytical system to determine their separation and retention times followed by $15 \mu \mathrm{L}$ injection of reaction mixture. The separations were achieved on a Supelco LC-18-BD, column $(250 \times 4.6 \mathrm{~mm}$ I.D., $5 \mu$ practical size, product number $58355 \mathrm{C} 40$, lot number 75251-06 (Supelco, distributed by SigmaAldrich, Brockville, ON, Canada) using a mobile phase comprising of A (water $+0.1 \%$ trifluoroacetic acid, v/v) and B acetonitrile. The mobile phase composition, delivered at $1.2 \mathrm{ml} / \mathrm{min}$, at a column thermostat temperature of $35{ }^{\circ} \mathrm{C}$, was $0-2 \min 15 \%$ $\mathrm{B}$ isocratic, $2-7 \min 15-40 \% \mathrm{~B}$. The column was washed for $3 \mathrm{~min}$ with $100 \% \mathrm{~B}$ for $3 \mathrm{~min}$ and equilibrated at initial composition for 5 min before next injection. The signals were monitored at 230 $\mathrm{nm}$ (band width 4, reference off) with DAD with the range of 200-400 $\mathrm{nm}$ and peak width of $0.1 \mathrm{~min}$. The mass spectrometric detection of target compounds was achieved with ESI operating in positive ionization mode within a scanning mass range of 100-700 amu. The optimized mass spectrometric conditions were: gas temp $350{ }^{\circ} \mathrm{C}$, drying gas (N2) flow $10 \mathrm{~L} / \mathrm{min}$, nebulizer pressure 50 bar, capillary voltage $3000 \mathrm{~V}$.

\section{Caco-2 Transwell incubation studies}

Caco-2 cells (cell line C2Bbe1) were cultivated in insert filters $\left(0.6 \mathrm{~cm}^{2}, 0.4 \mu \mathrm{m}\right.$ and $\left.12 \mathrm{~mm}\right)$ and placed in a 24-well plate with Dulbecco's modified Eagle's medium (DMEM) at $37^{\circ} \mathrm{C}, 5 \% \mathrm{CO}_{2}$ for approximately 21 days.

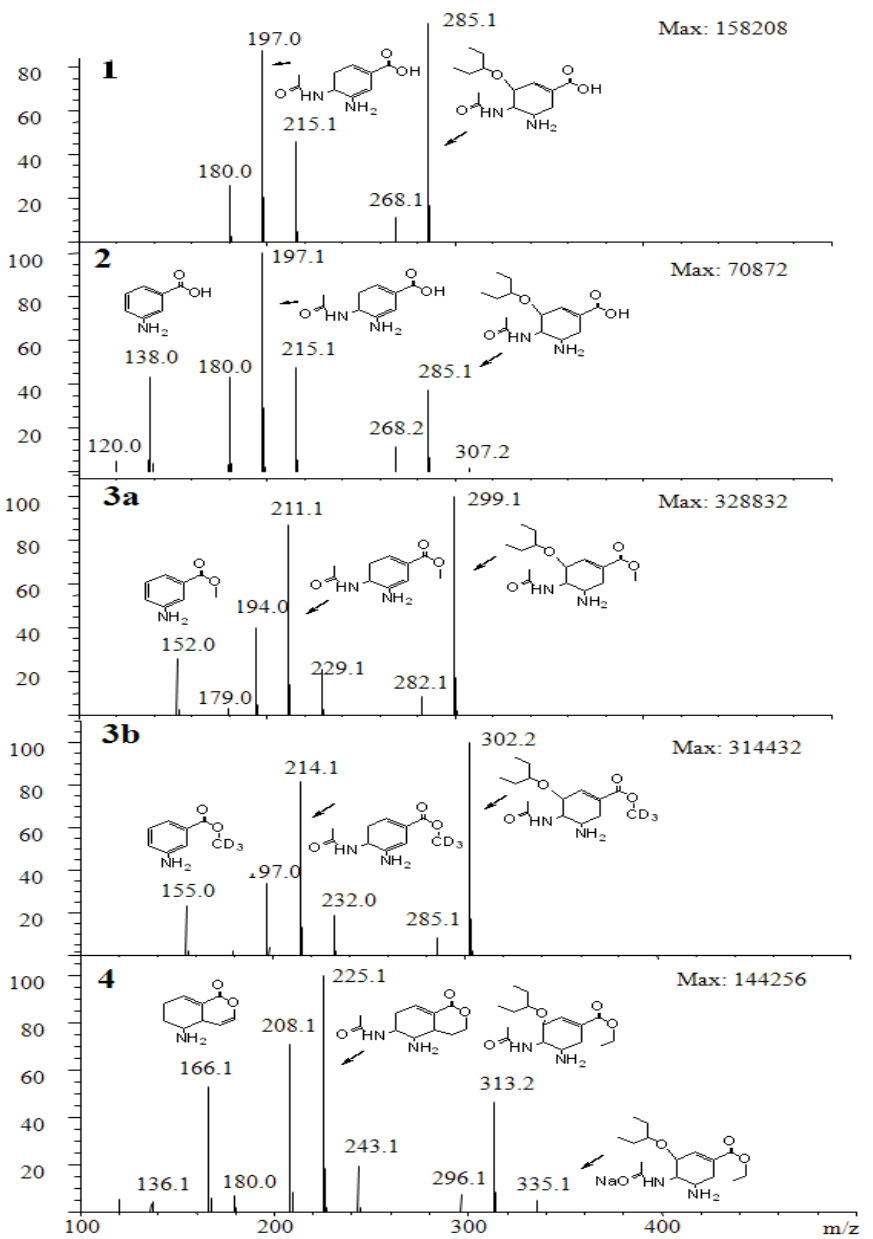

Figure 2. Mass spectra and structures of major fragments of oseltamivir phosphate (4), oseltamivir carboxylate (2), and the methanolic metabonates $3 a$ and $3 b$ (deuterated form of $3 a$ ) and an unknown isomer (1). The spectra are presented with mass range of $100-500 \mathrm{~m} / \mathrm{z}$ (x-axis) and relative abundance of fragments (y-axis). 
When cell electroconductivity exceeded 280 , the differentiated cells were ready for the transport study. Transport of oseltamivir was examined in both the apical to basal and basal to apical direction with $40 \mu \mathrm{L}$ of oseltamivir added to either the apical or basal chamber. For each test, $400 \mu \mathrm{L}$ of media was added into each insert or well. Two blank controls were arranged as pure cell culture media without inserts and cell culture media with drug. Three samples were tested AD01, Echinacea (NRP 451) and Panax quinquefolium extraxct (NRP 452) at a concentration $5 \mu \mathrm{L} / 400 \mu \mathrm{L}$ medium. The plates were then incubated at $37^{\circ} \mathrm{C}$ for $1 \mathrm{hr}$. Then the media in the inserts and wells were collected respectively and mixed immediately with $600 \mu \mathrm{L}$ stop solution. The samples were centrifuged and filtered prior to HPLC analysis.

\section{RESULTS}

Initial studies with methanolic stock solutions of oseltamivir phosphate and oseltamivir carboxylate. Figure 1 shows the HPLC-DAD profile of a mixture of authentic oseltamivir carboxylate (\#2) and its parent compound oseltamivir (\#4) eluting at respective retention times $6.8 \mathrm{~min}$ and $8.9 \mathrm{~min}$. The mass spectrometric fragmentation pattern of both compounds followed similar pattern in the positive electrospray ionization. In the positive ionization mode both showed the loss of $\mathrm{m} / \mathrm{z} 88$ (pentan-3-ol moiety) followed by the loss of $\mathrm{m} / \mathrm{z} 59$ (acetamide moiety) (Figures 2 - \#2 and 2 - \#4). However $[\mathrm{M}+\mathrm{Na}]^{+}$with an $\mathrm{m} / \mathrm{z}$ of 313 was also observed in case of 4.

HPLC analysis of a HLM incubation mixture with Tris buffer $\mathrm{pH} 7.4$ found the parent compound and a single large peak with a retention time between that of the carboxylate metabolite and parent compound (Figure 1C - \#3a). Mass spectral analysis of the major unknown peak (compound \#3a) found an $M+1$ of 299 (Figure 2 - \#3a). This mass was $\mathrm{m} / \mathrm{z}-14$ lower than the parent compound and higher by the same amount than the carboxylate. The mass spectral fragmentation profile was similar to that of the parent compound with the exception that all major ions had an m/z-14 mass suggesting formation of the carboxylate metabolite with subsequent methylation of the decarboxylated side chain. Incubation of an aliquot of the methanolic stock solutions of oseltamivir or the carboxylate in denatured HLM did not result in the formation of this peak. The peak was also absent after overnight incubation of oseltamivir or carboxylate with methanol in reaction buffer at $37^{\circ} \mathrm{C}$. The initial LC-MS sample at room temperature in a light protected injection vial was re-run after a 3 week period and only a single peak was detected with a mass and retention time identical to that of the methyl derivative. When deuterated methanol was used in the HLM incubation, an M+1 of 302 (Figure 1D - \#3b) was detected. The three extra mass was from the deuterium methyl group which confirmed that the product (\#3b) was formed by methylation of the decarboxylated side chain.

In order to check the effects of different solvents the incubation was then repeated with acetonitrile, ethyl acetate, $95 \%$ ethanol, and methanol compared to an aqueous control sample (Figure 3). EtOH $(0.25 \%)$ had the least inhibitory effect.

Oseltamivir was then prepared as an aqueous stock solution. In the HLM incubation matrix (Tris, $\mathrm{pH} 7.5$ ) a new peak with a retention time of $6.5 \mathrm{~min}$ that eluted prior to authentic carboxylate was detected (Figure 1B) with the corresponding decrease in the intensity of 4 which indicate the conversion of 4 into 1 (non-quantitative observation). Its mass spectral profile was similar to 2 but with the absence of an amino-benzoic acid moiety with mass fragment of $\mathrm{m} / \mathrm{z} 138$ (Fig 2-1). Additionally, in the case of 1 , the protonated molecular ion had a relative abundance of 100 compared to 2 in which this mass had a relative abundance of 40 , suggesting conversion of 2 into a more stable form.

Oseltamivir did not inhibit the cytochrome P450-mediated metabolism of the CYP3A4 (DBF) and CYP2C (BFC) marker substances in the fluorometric plate reader assay.

\section{Natural health products}

Testing was then extended to extracts of different natural products using $20 \mathrm{mg} / \mathrm{mL}$ stock solutions in $95 \% \mathrm{EtOH}$ or water. The ethanolic extracts were diluted to $1 \mathrm{mg} / \mathrm{mL}$ with water to reduce the solvent effect. The aqueous extracts were examined directly. Neither of the NRP 452 extracts affected formation of the carboxylate (Figure 4). The ethanolic extract of Echinacea had no effect but the aqueous solution reduced formation of the 
carboxylate and unknown $\# 1$ by about $18 \%$. Ethanolic extracts of ground Cree plant material were then examined as above for the formation of the carboxylate (Figure 5). Of the 17 samples examined, 6 (AD01, 02, 07, 09; W1, 2) reduced formation of the carboxylate by greater than $20 \%$. Three of these extracts (AD01, 02 and W1) had an inhibitory effect of about 40 to $55 \%$. Another 6 products (AD03, 11; W4, 6, 8, 9) reduced formation by about 10 to $20 \%$. A similar pattern was observed when the effect on formation of the carboxylate and metabolite \#3 was examined. AD01 and W1 and W4 were then prepared in the traditional manner and aliquots of these preparations taken at 2 time points were not inhibitory (Figure 6).

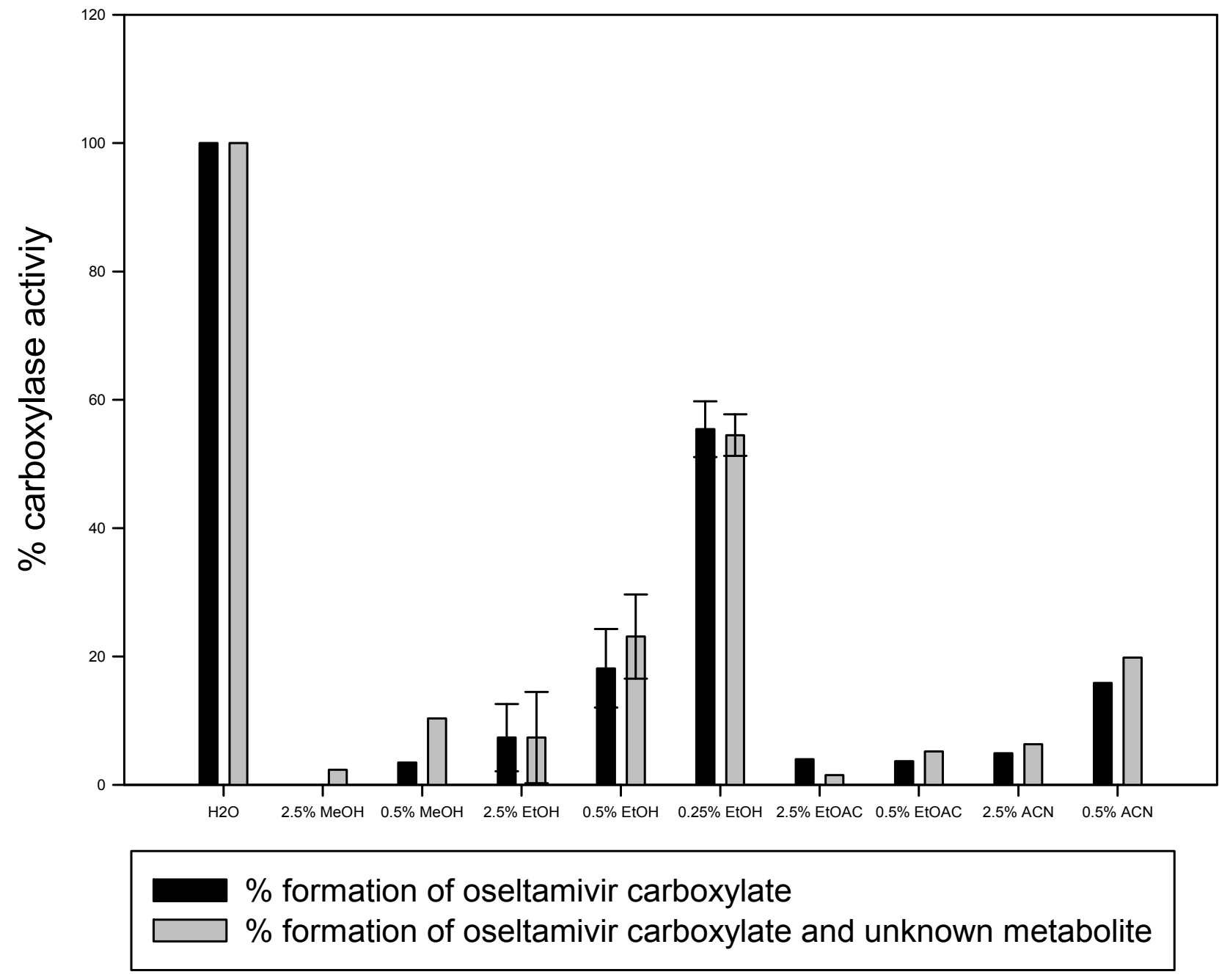

Figure 3. A comparative study of the effect of methanol (MeOH), ethanol (EtOH), ethyl acetate (EtOAC) and acetonitrile $(\mathrm{ACN})$ on the formation of the active oseltamivir carboxylate alone or together with an unknown metabolite relative to the aqueous control by the human liver microsome incubation mixture in Tris buffer $\mathrm{pH} 7.4 ; \mathrm{n}=1-3$, mean $\pm \mathrm{SEM}$. 


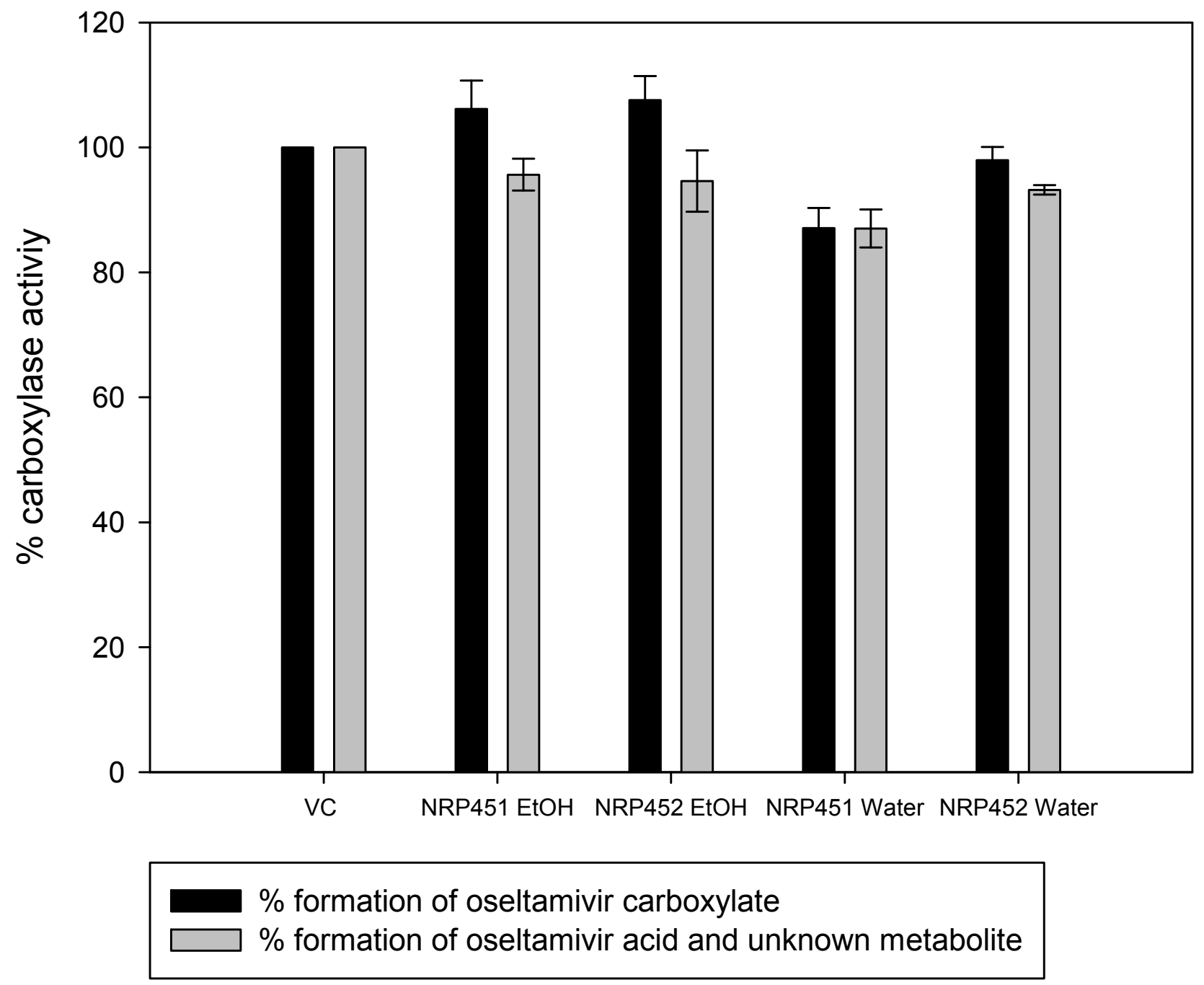

Figure 4. A comparative study of the effect of aqueous and ethanolic extracts of an Echinacea (NRP 451) and Panax quinquefolium extract (NRP 452) on the formation on the formation of the active carboxylate alone or together with an unknown metabolite relate to the aqueous and ethanolic vehicle control (VC) by the human liver microsome incubation mixture in Tris buffer $\mathrm{pH}$ 7.4. Ethanolic extracts were made at $20 \mathrm{mg} / \mathrm{mL}$ in $95 \% \mathrm{EtOH}$, and then diluted with water, the final concentration in the incubation matrix was $2.5 \mu \mathrm{g} / \mathrm{mL}$. Aqueous extracts were made at $20 \mathrm{mg} / \mathrm{mL}$ in water; the final concentration in the incubation matrix was $50 \mu \mathrm{g} / \mathrm{mL} . \mathrm{n}=3$, mean $\pm \mathrm{SEM}$.

Three traditional Chinese medicines, Chrysanthemun Flower (NRP 265), Du Huo (NRP 267) and Chai $\mathrm{Hu}$ (NRP 269) were examined at low concentrations as these are traditional preparations that would be used by individuals with respiratory problems. Of these 3 products, NRP 265 and 267 were not inhibitory and the third, NRP 269 had minimal inhibitory activity under these test conditions (Figure 7). Goldenseal had strong inhibitory activity and reduced carboxylate formation by about $75 \%$ (Figure 7).

Caco-2 cell mediated transport was examined (Table 2). Oseltamivir readily moved between the apical and basal chambers, preferentially in the apical to basal direction. Of the 3 products examined for an interaction, AD01, NRP 451 and 452 , only NRP 452 affected the apical to basal movement of oseltamivir. 


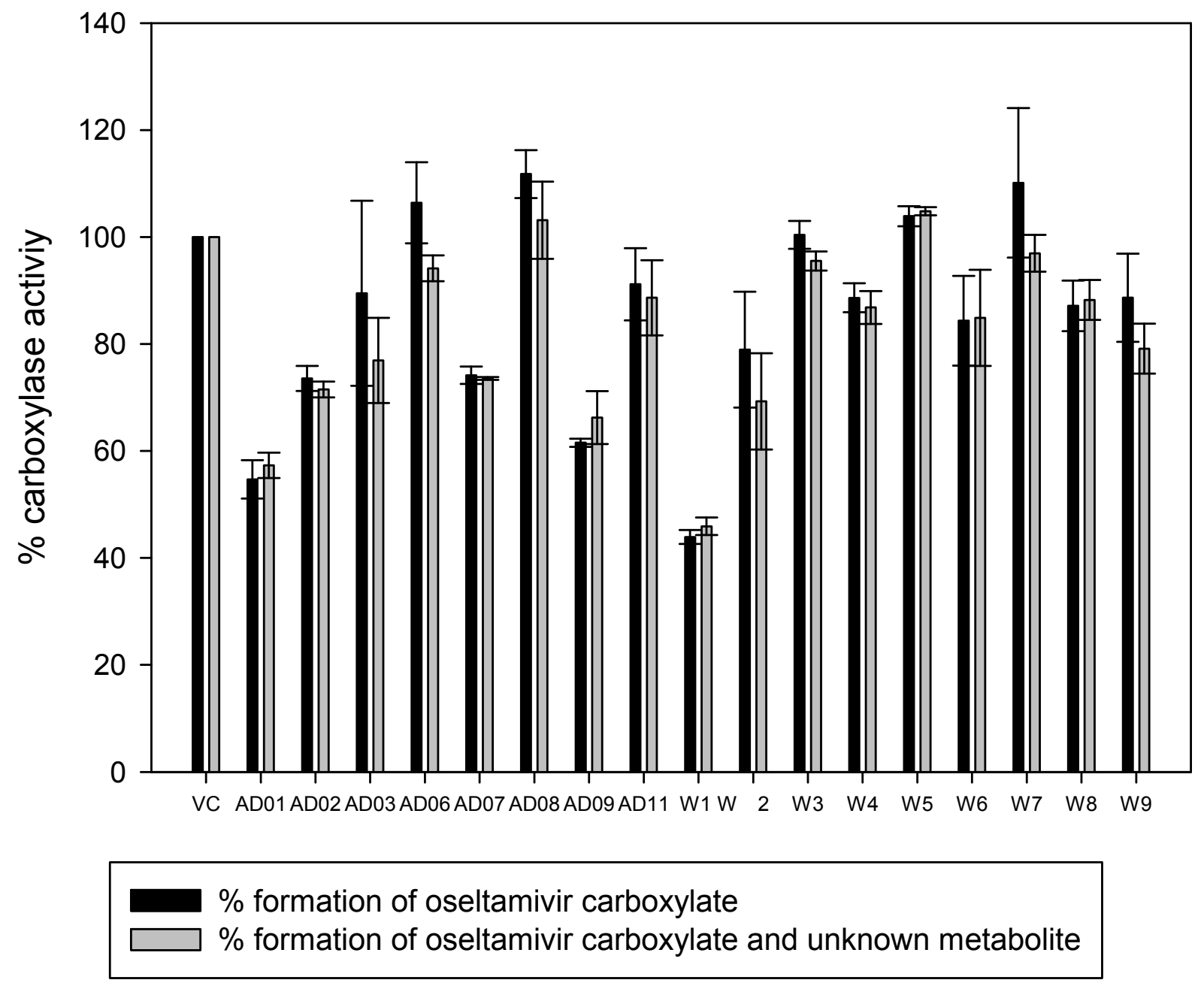

Figure 5. A comparative study of the effect of several ethanolic extracts of ground plant material on the formation of the active carboxylate alone or together with an unknown metabolite relative to the ethanolic vehicle (V) control by the human liver microsome incubation mixture in Tris buffer $\mathrm{pH} 7.4$. Ethanolic extracts were made at $20 \mathrm{mg} / \mathrm{mL}$ in $95 \% \mathrm{EtOH}$, and then diluted with water to the final concentration in the incubation matrix of $2.5 \mu \mathrm{g} / \mathrm{mL} . \mathrm{n}=3, \mathrm{mean} \pm \mathrm{SEM}$.

The affect was less pronounced in the basal to apical direction. The findings however are somewhat confounded by the relative amounts of the metabolites also present.

\section{DISCUSSION}

Herb-drug, food-drug, nutrient-drug, and drug-drug interactions are known to cause adverse events that can seriously impact patient morbidity and mortality (14). The findings in this study would suggest that extracts from several different botanical products, both commercial and traditional medicines, have the potential to reduce the formation of the active carboxylate product. Our study observed that minor differences in incubation conditions can markedly affect the metabolic profile. The findings have been confounded however by the detection of three previously unreported metabolites. Although compounds 1 and 3a have similar chromatographic properties to the rat metabolites reported by Sweeny et al. (9), their mass spectral properties are markedly different. 


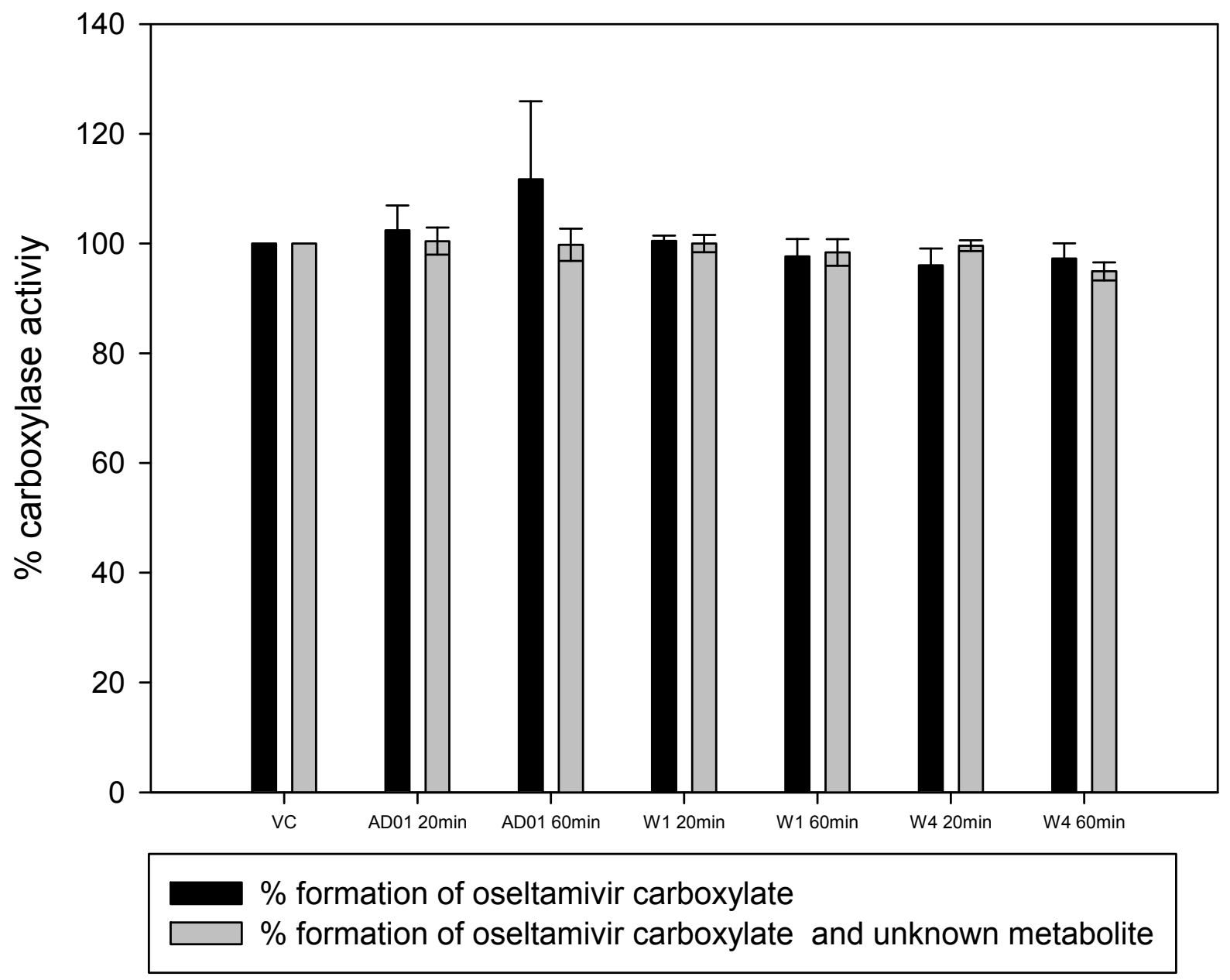

Figure 6. A comparative evaluation of the effect of aliquots from traditionally prepared AD01, W1 and W4 teas on the formation of the active carboxylate alone or together with an unknown metabolite relative to the aqueous vehicle control (VC) where $1 \mathrm{gm}$ of dried material was boiled in $250 \mathrm{~mL}$ water for $20 \mathrm{~min}$ or $60 \mathrm{~min}$. Aliquots were taken and then analyzed by HPLC. $\mathrm{n}=3$ mean \pm SEM.

Table 2. Ratio of the amounts of oseltamivir present in the apical and basal chambers in the Caco-2 transport study where $40 \mu \mathrm{L}$ of aqueous $10 \mathrm{mg} / \mathrm{mL}$ oseltamivir added to either the apical or basal chamber and incubated for $1 \mathrm{hr}$. ( $\mathrm{n}=1$ ).

\begin{tabular}{ccccc} 
Chambers & Blank control & AD01 & NRP 451 & NRP 452 \\
\hline Apical to Basal & 0.29 & Oseltamivir & 0.19 \\
Basal to Apical & 0.25 & 0.29 & 0.25 & 0.33 \\
& & 0.30 & 0.29 & 0.15 \\
\hline Apical to Basal & 0.29 & Total of all peaks & 0.26 \\
Basal to Apical & 0.25 & 0.29 & 0.31 & 0.35 \\
\hline
\end{tabular}




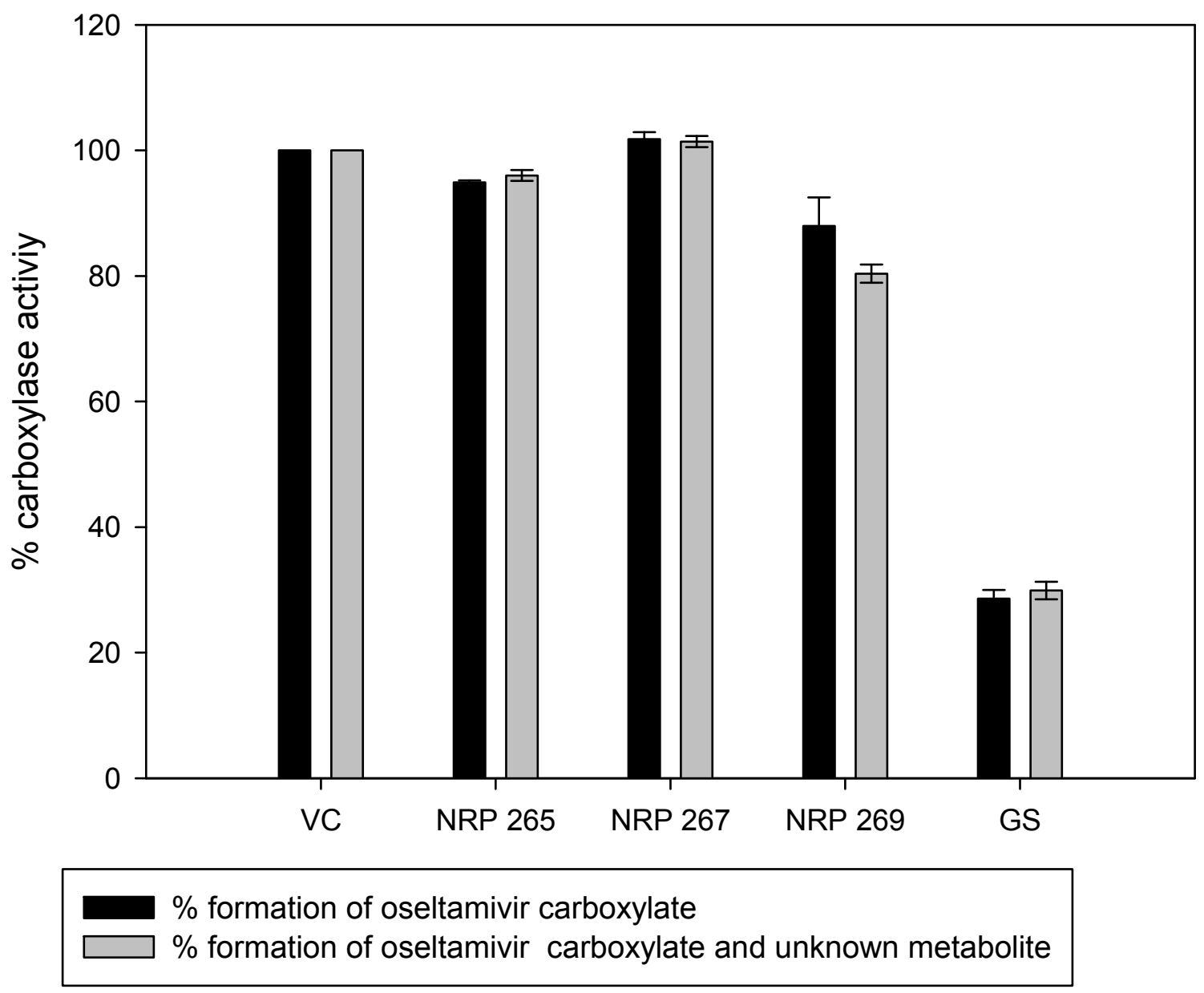

Figure 7. A comparative study of the effect of extracts from Goldenseal (GS, NRP 273); and Chrysanthemun Flower (NRP 265), Du Huo (NRP 267) and Chai Hu (NRP 269) medicinal granules on the formation of the active oseltamivir carboxylate alone or together with an unknown metabolite relative to the vehicle control (VC) by the human liver microsome incubation mixture in Tris buffer $\mathrm{pH} 7.4, \mathrm{n}=3$, mean \pm SEM.

The major peak obtained when oseltamivir was incubated with active but not denatured HLM in the presence of $\mathrm{MeOH}$ may be a metabonate resulting from condensation or trans-esterification of the carboxylate with methanol to give the corresponding methyl-ester. This was confirmed by the $\mathrm{CD}_{3} \mathrm{OD}$ study suggesting that in addition to a slow chemical conversion, trans-esterification is also be mediated in HLM by an enzyme such as Omethytransferase. Further studies are required to determine if the carboxylate can be trans-esterified and if these mechanisms could proceed with other small chain alcohols as the donor.
The extracts from the Cree botanicals were prepared under identical conditions allowing a comparative evaluation of their potential to affect this enzyme. The findings in this study can be grouped into poor, fair and moderate to strong potential for interacting with this enzyme. It is noteworthy however than when 3 of these traditional medical botanicals were made as infusions (teas) they did not affect the formation of the active carboxylate product. As this initial screen looked at low concentrations of a single Echinacea product and only 3 traditional Chinese medicines, the findings can only suggest that there may be a potential for Echinacea and Chai Hu (NRP 269) to 
interact. Interestingly, Goldenseal had a very strong inhibitory effect.

The initial transport studies reported here suggest that $P$. quinquefolium extract (NRP 452) may interfere with mediated transport through the Caco-2 cells. The current system does not provide mechanistic information. Normally, use of these cells examines transport by the P-glycoprotein efflux transporter; however, peptide transporter 1 is known to transport oseltamivir along with $\beta$-lactam antibiotics, some anti-viral and anti-cancer agents. The presence of the active carboxylate drug and compounds \#1 and 3a confounds the understanding of these results but does justify further evaluation.

These findings would then suggest that under some conditions, several of these medical botanicals could cause an adverse interaction but additional studies are required to extend and clarify the significance of these findings. As with many medications, the actual amount and the rate that the medicine is consumed will affect the response. Oseltamivir, as reported in the literature, did not inhibit the cytochrome P450-mediated metabolism of the two marker substances, DBF (CYP3A4/5/7 and 19) and BFC (CYP2C) in the fluorometric plate reader assay but additional studies should be undertaken to look at a wider range of isozymes that may shed mechanistic information on the solvent effects and formation of the 3 unknowns. As this study has demonstrated that natural products examined have the in vitro potential to mediate the formation of the carboxylate, further studies are warranted with a wider range of products. As the consequence of developing resistance to this antiviral agent posses a severe risk, clinical evaluation of these findings are warranted to determine if there is an interaction with the absorbed fraction which may differ from the herbal extract.

\section{ACKNOWLEDGEMENTS}

Very special thanks are due to the Cree Nation of Mistissini and Whapmagoostui First Nations as well as to 54 Cree Elders of both nations who kindly agreed to be interviewed. They made this article possible by allowing us to use, for the purposes of this research, their knowledge relating to medicinal plants, transmitted to them by their elders. Their trust has also enabled a useful exchange between indigenous knowledge and western science.

\section{REFERENCES}

1. Shi, D., Yang, J., Yang, D., LeCluyse, E.L., Black, C., You, L., Akhlaghi, F., Yan, B. Anti-influenza prodrug oseltamivir is activated by carboxylesterase human carboxylesterase 1 , and the activation is inhibited by antiplatelet agent clopidogrel. J Pharmacol Exp Ther, 319:1477-1484, 2006.

2. Yang, D., Pearce, R.E., Wang, X., Gaedigk, R., Wan, Y.J., Yan, B. Human carboxylesterases HCE1 and HCE2: ontogenic expression, inter-individual variability and differential hydrolysis of oseltamivir, aspirin, deltamethrin and permethrin. Biochem Pharmacol, 77:238-247, 2009.

3. Zhu, H.J., Patrick, K.S., Yuan, H.J., Wang, J.S., Donovan, J.L., DeVane, C.L., Malcolm, R., Johnson, J.A., Youngblood, G.L., Sweet, D.H., Langaee, T.Y., Markowitz, J.S. Two CES1 gene mutations lead to dysfunctional carboxylesterase 1 activity in man: clinical significance and molecular basis. Am J Hum Genet, 82:1241-1248, 2008.

4. He, G., Massarella, J., Ward, P. Clinical pharmacokinetics of the prodrug Oseltamivir and its active metabolite Ro 64-0802. Clin Pharmacokinet, 37:471-484, 1999.

5. Morimoto, K., Nakakariya, M., Shirasaka, Y., Kakinuma, C., Fujita, T., Tamai, I., Ogihara, T. Oseltamivir (Tamiflu) efflux transport at the bloodbrain barrier via P-glycoprotein. Drug Metab Dispos, 36:6-9, 2008.

6. Ogihara, T., Kano, T., Wagatsuma, T., Wada, S., Yabuuchi, H., Enomoto, S., Morimoto, K., Shirasaka, Y., Kobayashi, S., Tamai, I. Oseltamivir $\left(\right.$ Tamiflu $^{\mathrm{TM}}$ ) is a substrate of PEPT1. Drug Metab Dispos. 37:1676-1681, 2009.

7. Zhu, H.J., Markowitz, J.S. Activation of the antiviral prodrug oseltamivir is impaired by two newly identified carboxylesterase 1 variants. Drug Metab Dispos, 37(2):264-267, 2009.

8. Massarella, J.W., He, G.., Dorr, A., Nieforth, K., Ward, P., Brown, A. The pharmacokinetics and tolerability of the oral neuraminidase inhibitor oseltamivir (Ro 64-0796/GS4104) in healthy adult and elderly volunteers. J Clin Pharmacol, 40:836$843,2000$.

9. Sweeny, D.J., Lynch, G., Bidgood, A.M., Lew, W., Wang, K.Y., Cundy, K.C. Metabolism of the influenza neuraminidase inhibitor prodrug oseltamivir in the rat. Drug Metab Dispos, 28:737$741,2000$.

10. Zhu, H.J., Appel, D.I., Johnson, J.A., Chavin, K.D., Markowitz, J.S. Role of carboxylesterase 1 and impact of natural genetic variants on the hydrolysis of trandolapril. Biochem Pharmacol, 77:1266-1272, 2009. 
11. Zhu, H.J., Appel, D., Jiang, Y., Markowitz, J.S. Age and sex-related expression and activity of carboxylesterase 1 and 2 in mouse and human liver. Drug Metab Dispos. 7:1819-1825, 2009.

12. Ose, A., Kusuhara, H., Yamatsugu, K., Kanai, M., Shibasaki, M., Fujita, T., Yamamoto, A., Sugiyama, Y. P-glycoprotein restricts the penetration of oseltamivir across the blood-brain barrier. Drug Metab Dispos, 36:427-434, 2008.

13. Izumi, Y., Tokuda, K., O'Dell, K., Zorumski, C., Narahashi, T. Synaptic and behavioral interactions of oseltamivir (Tamiflu) with neurostimulants. Hum Exp Toxicol, 27:911-9117, 2008.

14. Sørensen, J.M., Herb-therapeutic product, foodtherapeutic product, nutrient-therapeutic product, and therapeutic product-therapeutic product interactions: mechanisms involved and their medical implications. J Altern Complement Med, 8:293-308, 2002.

15. Leduc, C., Coonishish, J., Haddad, P.S., and Cuerrier, A. Plants used by the Cree nation of Eeyou Istchee (Quebec, Canada) for the treatment of diabetes: a novel approach in quantitative Ethnobotany. J Ethnopharmacol. 105:55-63, 2006.

16. Spoor, D. C. A., Martineau, L. C., Leduc, C., Benhaddou-Andaloussi, A., Meddah, B., Harris, C., et al. Selected plant species from the Cree pharmacopoeia of northern Quebec possess antidiabetic potential. Can J Physiol Pharmacol. 84, 847-858, 2006. 